\title{
Preincubation with the Proteasome Inhibitor MG-132 Enhances Proteasome Activity via the Nrf2 Transcription Factor in Aging Human Skin Fibroblasts
}

\author{
DAVID CHRISTIAN KRAFT,${ }^{a}$ CUSTER C. DEOCARIS,${ }^{b, c}$ \\ RENU WADHWA, ${ }^{b}$ AND SURESH I. S. RATTAN ${ }^{a}$ \\ ${ }^{a}$ Laboratory of Cellular Ageing, Danish Centre for Molecular Gerontology, \\ Department of Molecular Biology, University of Aarhus, Aarhus, Denmark \\ ${ }^{b}$ Gene Function Research Center, National Institute of Advanced Industrial \\ Science and Technology (AIST), 1-1-1 Higashi, Tsukuba, Japan \\ ${ }^{c}$ Cancer Research and Radiation Biology Laboratory, Philippine Nuclear \\ Research Institute, Quezon City, Philippines
}

\begin{abstract}
Strategies that lead to the upregulation of the proteasome are known to elicit beneficial consequences to the organism by countering oxidative stress-associated disorders, such as protein conformational diseases, cancer, and aging. Mild treatment with proteasome inhibitors has been previously demonstrated to stimulate proteasome activity and cellular resistance against oxidative injury. However, the mechanism for this action has not been clearly defined. We examined the role of the nuclear factor-E2-related factor 2 (Nrf2) in fibroblasts, a key transactivator of the antioxidant response pathway, in the regulation of the proteasome by its inhibitor MG-132. Here, we demonstrate that the stimulation of the proteasome by low levels of MG-132 can be abrogated by small interfering RNAs (siRNAs) targeted against Nrf2. Consistently, cells that constitutively express Nrf2 exhibit elevated levels of proteasome activities. We further investigate how its beneficial effects, that is, proteasome stimulation, are manifested in young and replicative-senescent cells. Our data underscore that manipulation of $\mathrm{Nrf} 2$ by the administration of pharmacologically low levels of proteasome inhibitors may prove to be an alternatively potent strategy for inducing long-term protective effects against oxidative stress.
\end{abstract}

KEYWORDS: Nrf2; proteasome; MG-132; fibroblast; hormesis; aging

Address for correspondence: Dr. Suresh I.S. Rattan, Department of Molecular Biology, University of Aarhus, Gustav Wieds Vej 10C, DK8000 Aarhus-C, Denmark. Voice: +458942 5034; fax: +45 86123178.

e-mail: rattan@mb.au.dk

Ann. N.Y. Acad. Sci. 1067: 420-424 (2006). (C) 2006 New York Academy of Sciences. doi: 10.1196/annals.1354.060 


\section{INTRODUCTION}

Long-term exposure to proteasome inhibitors leads to severe oxidative stress, cell cycle arrest, downregulation of the proteasome, accumulation of protein aggregates, and appearance of senescence-like phenotypes. ${ }^{1-5}$ It is widely accepted that the induction of cell cycle arrest is mainly attributed to the inhibition of the ubiquitin $26 \mathrm{~S}$ proteasome pathway, which controls cell cycle regulators. ${ }^{5,8}$ Analysis of gene expression in neural cells subjected to 12 -week proteasome inhibition showed a wide range of cellular changes, such as reduced proteasome expression, and a weakened oxidative defense mechanism accompanied by the downregulation of glutathione S-transferase among others. ${ }^{6}$ Such reduction in oxidative defense in response to proteasome inhibition is also evident from the increased levels of DNA and RNA oxidation. ${ }^{7}$

Short-term proteasome inhibition, on the other hand, results in an opposing effect that leads to improved cellular fitness accompanied by the induction of glutathione S-transferase (GST), heat-shock proteins (HSPs), and several proteasome genes. ${ }^{9}$ The activation of oxidative-defense genes is known to occur through transcription factors like heat-shock factor 1 (HSF1), activator protein 1 (AP-1), and Nrf2. ${ }^{9,10}$ Recently, it has been found that the proteasome is also transcriptionally regulated by Nrf2, suggesting a role for a proteasomal degradation pathway in the cellular adaptive response to oxidative stress. ${ }^{11} \mathrm{In}$ fact, the promoter regions of $20 \mathrm{~S}$ and 19S, but not the immunoproteasome, harbor two antioxidant response elements (AREs). ${ }^{12}$

The Nrf2 is a cap-n-collar family of transcription factors that regulates cellular defenses against ROS. Under normal physiologic conditions, it exists in its inactive form because of sequestration by the cytoplasmic nuclear translocation-inhibitor KEAP1, which delivers it to proteasomal degradation. ${ }^{13-15}$ Upon oxidative stress, Nrf2 is released from KEAP1 and translocates to the nucleus and initiates transcription of some 200 genes with the cis-acting antioxidant response element (ARE). Most of these genes have known roles in protecting the cell against oxidative and electrophilic stressors. ${ }^{11}$ As activity of the proteasome is shown to be stimulated by short-term or low-dose exposure to proteasome inhibitors, we wanted to test whether Nrf2 is the transcription factor that controls this negative feedback loop of proteasome activity and gene expression. Furthermore, we tested whether the response to proteasome inhibition differs between young and replicative-senescent human cells.

\section{RESULTS AND DISCUSSION}

Normal adult human fibroblasts were co-transfected with the pNQR-ARE, a firefly luciferase reporter construct controlled by an ARE in the promoter region, and pRL-RSV, a constitutive Renilla luciferase expression plasmid. After incubation for $12 \mathrm{~h}$ with increasing amounts of MG-132 (2.5-30 nM), 
(A)

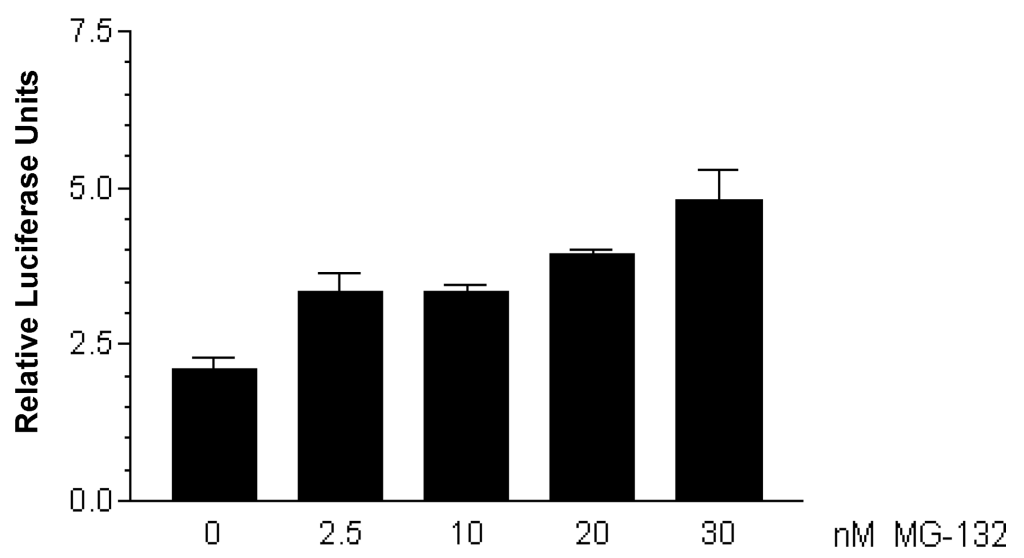

(B)

MG-132 (30nM)

pUC-Nrf2i

PCDNA3-Nrf2

pUC vector

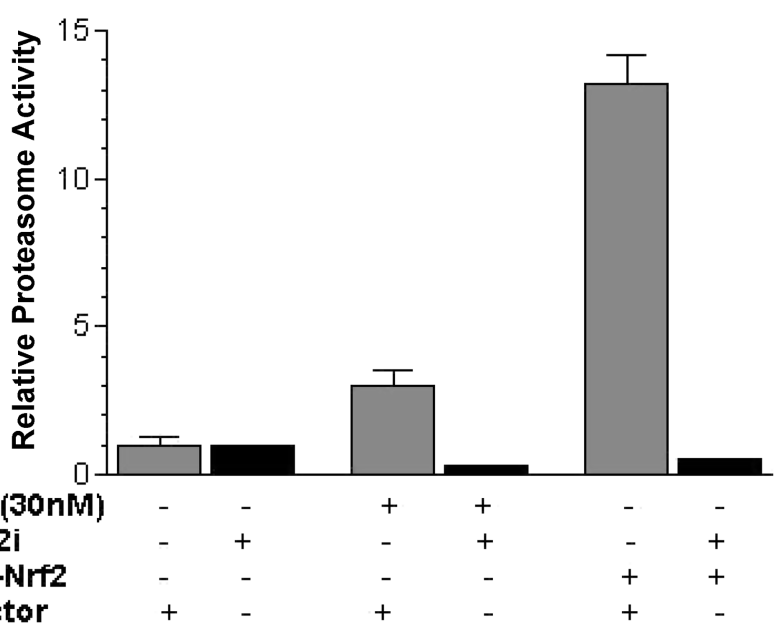

FIGURE 1. Pretreatment with the proteasome-inhibitor MG-132 leads to an increased $\mathrm{Nrf2}$, an upregulated proteasome activity in young cells, which was abolished by Nrf2 siRNA. (A) Dual-luciferase reporter (pNQR-ARE, pRL-RSV) on TIG-1 cells treated with different concentrations of the MG-132 for 12-16 h resulted in the upregulation of the Nrf2. (B) Assay for the chymotrypsin-like proteasome activity of young cells after treating with $30 \mathrm{nM} \mathrm{MG}-132$ for $16 \mathrm{~h}$ and Nrf2 shRNA.

a dual-luciferase assay was performed using a commercial kit from Promega (Madison, WI). The increasing levels of MG-132 resulted in a dose-dependent elevation of Nrf2 activity with the highest induction at $30 \mathrm{nM}$ (FIG. 1A). After $12 \mathrm{~h}$ of incubation with $30 \mathrm{nM}$ MG-132, proteasome activity was increased but not to the extent of the cells transfected with the pcDNA3-Nrf2, a 
CMV-driven Nrf2 expression cassette (FIG. 1B). The increase in proteasome activity in Nrf2-overexpressing transfectants and in cells treated with $30 \mathrm{nM}$ MG-132 was abolished after (co-)transfection with pUC-Nrf2i, a U6 promoterdriven short hairpin RNA against Nrf2 (FIG. 1B). These results demonstrated that proteasome activity could be stimulated by MG-132 and this proteasomestimulatory effect may be controlled by the Nrf2 transcription factor.

Finally, we tested the reactivity of young and replicative-senescent cells to mild and severe treatment with MG-132. Proteasome activity was induced by $30 \mathrm{nM}$ (mild) MG-132 treatment both in replicative-senescent (old) and young cells. However, in the old cells induction was only about one-third of the increase in proteasome activity exhibited by young cells. Treatment with MG-132 (30 $\mu \mathrm{M}$ for $1 \mathrm{~h})$ induced severe cell death in the rapidly growing young cells $(\sim 84 \%$ death), whereas the replicative-senescent cells were less affected ( $\sim 33 \%$ death) (data not shown). These data suggest that MG-132induced protective effects against oxidative stressors via Nrf2 activation may be differentially regulated in young and senescent cells. ${ }^{16-19}$

In summary, our data underscore that the manipulation of the Nrf2 pathway by pharmacologically low levels of proteasome inhibitors could prove to be an alternative potent strategy for inducing long-term protective effects against oxidative stress and aging. We are further investigating how such beneficial effects, that is, proteasome stimulation and increased fitness, are manifested during replicative senescence.

\section{ACKNOWLEDGMENTS}

We acknowledge the support of the Gene Function Research Laboratory, National Institute for Advanced Industrial Science and Technology (AIST), for travel and research fellowship to David Christian Kraft, and Professor Masayuki Yamamoto, Tara Institute, University of Tsukuba, Japan for providing the Nrf2 expression and reporter plasmids, and for helpful technical discussion. The Laboratory of Cellular Ageing (University of Aarhus) is supported by research grants from the Danish Research Councils, Carlsberg Fund, Senetek PLC, and EU's Biomed Health Programmes.

\section{REFERENCES}

1. YAno, M. et al. 2005. Chaperone activities of the $26 \mathrm{~S}$ and $20 \mathrm{~S}$ proteasome. Curr. Protein Pept. Sci. 6: 197-203.

2. Bajorek, M., D. Finley \& M.H. Glickman. 2003. Proteasome disassembly and downregulation is correlated with viability during stationary phase. Curr. Biol. 13: $1140-1144$.

3. DING, Q. et al. 2003. Characterization of chronic low-level proteasome inhibition on neural homeostasis. J. Neurochem. 86: 489-497. 
4. Chondrogianni, N. et al. 2003. Central role of the proteasome in senescence and survival of human fibroblasts: induction of a senescence-like phenotype upon its inhibition and resistance to stress upon its activation. J. Biol. Chem. 278: 28026-28037.

5. YIN, D. et al. 2004. Proteasome inhibitor PS-341 causes cell growth arrest and apoptosis in human glioblastoma multiforme (GBM). Oncogene 24: 344-354.

6. DING, Q. et al. 2004. Analysis of gene expression in neural cells subject to chronic proteasome inhibition. Free Radic. Biol. Med. 36: 445-455.

7. DING, Q. et al. 2004. Proteasome inhibition increases DNA and RNA oxidation in astrocyte and neuron cultures. J. Neurochem. 91: 1211-1218.

8. IMAI, J. et al. 2003. The molecular chaperone Hsp90 plays a role in the assembly and maintenance of the 26S proteasome. EMBO J. 22: 3557-3567.

9. KIM, D., S.H. KIM \& G.C. LI. 1999. Proteasome inhibitors MG132 and lactacystin hyperphosphorylate HSF1 and induce hsp70 and hsp27 expression. Biochem. Biophys. Res. Commun. 254: 264-268.

10. TACCHINI, L. et al. 2001. Influence of proteasome and redox state on heat shockinduced activation of stress kinases, AP-1 and HSF. Biochim. Biophys. Acta 1538: 76-89.

11. KWAK, M.K. et al. 2003. Modulation of gene expression by cancer chemopreventive dithiolethiones through the Keap1-Nrf2 pathway: identification of novel gene clusters for cell survival. J. Biol. Chem. 278: 8135-8145.

12. KWAK, M.K. et al. 2003. Antioxidants enhance mammalian proteasome expression through the Keap1-Nrf2 signaling pathway. Mol. Cell Biol. 23: 8786-8794.

13. MoI, P. et al. 1994. Isolation of NF-E2-related factor 2 (Nrf2), a NF-E2-like basic leucine zipper transcriptional activator that binds to the tandem NF-E2/AP1 repeat of the beta-globin locus control region. Proc. Natl. Acad. Sci. USA 91: 9926-9930.

14. Ітон, K. et al. 1999. Keap1 represses nuclear activation of antioxidant responsive elements by Nrf2 through binding to the amino-terminal Neh2 domain. Genes Dev. 13: 76-86.

15. Dinkova-Kostova, A.T. et al. 2002. Direct evidence that sulfhydryl groups of Keap1 are the sensors regulating induction of phase 2 enzymes that protect against carcinogens and oxidants. Proc. Natl. Acad. Sci. USA 99: 11908-11913.

16. LEE, C.S. et al. 2004. A proteasomal stress response: pre-treatment with proteasome inhibitors increases proteasome activity and reduces neuronal vulnerability to oxidative injury. J. Neurochem. 91: 996-1006.

17. LI, J. et al. 2005. Stabilization of Nrf2 by tBHQ confers protection against oxidative stress-induced cell death in human neural stem cells. Toxicol. Sci. 83: 313-328.

18. WAKABAYASHI, N. et al. 2004. Protection against electrophile and oxidant stress by induction of the phase 2 response: fate of cysteines of the Keap1 sensor modified by inducers. Proc. Natl. Acad. Sci. USA 101: 2040-2045.

19. Мотоналнi, H. \& M. Yамамото. 2004. Nrf2-Keap1 defines a physiologically important stress response mechanism. Trends Mol. Med. 10: 549-557. 